\title{
La tercera edad: el derecho al aprendizaje a lo largo de la
} vida

\section{Senior Citizens: The Right to Lifelong Learning}

Idosos: direito à aprendizagem ao longo da vida

Jaqueline Guadalupe Guerrero Ceh

Universidad Autónoma de Campeche, México

jgguerre@uacam.mx

https://orcid.org/0000-0002-2913-1309

\section{Resumen}

La edad no debe ser considerada como elemento discriminatorio para negar el acceso a la educación a personas gerontolescentes y ancianas. El conocimiento es parte de los derechos humanos: no deben existir barreras infundadas que impidan a nadie continuar a lo largo de su vida su desarrollo en un ambiente escolar. Este estudio evalúa las oportunidades educativas que tienen las personas mayores. Se trata de una investigación de tipo documental. Se analizó el marco internacional y nacional que respalda a las personas de edad; también se realizó un análisis del contexto actual de las oportunidades educativas a favor de este sector en México; y, por último, se concibió una propuesta de acción que permita la inclusión de la población mayor en el ámbito educativo. A través de la educación, las personas mayores se mantienen activas física y mentalmente; desarrollan un sentido de utilidad y pertenencia tanto familiar como socialmente. Sin duda es un error tratarlas como individuos pasivos, sin aportación; como se trata de una postura común en las familias, es importante actuar para erradicar esta conducta. Las personas mayores tienen un cúmulo de conocimientos y experiencia por aprovechar, son activas, productivas, que pueden ser 


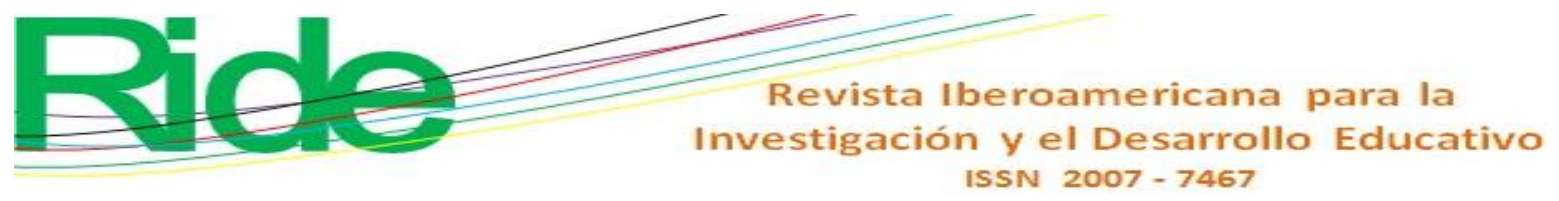

incorporadas a empresas y escuelas a través de programas destinados a cuidar su participación en estos espacios y formar un ambiente dignificador.

Palabras clave: derechos humanos, educación a lo largo de la vida, persona mayor, visibilidad social.

\begin{abstract}
Age should not be considered as a discriminatory element to close access to education for gerontolescents and the elderly. Knowledge is part of human rights and people should be allowed to continue attending school throughout their lives. This study answers the question: What are the educational opportunities that gerontolescents have? It is a documentary research. The international and national framework that supports the elderly was analyzed; an analysis of the current context of educational opportunities in favor of gerontolescents in Mexico was also carried out; finally, a proposal for action that allows the inclusion of people of age in the educational field was conceived. Through education, older people maintain their physical and mental activity, feeling useful and part of society, maintaining visibility in family and society. They are active, productive people that must be incorporated into companies and schools through programs aimed at caring for the participation of gerontolescents in these spaces, in a dignifying environment.
\end{abstract}

Keywords: human rights, lifelong education, elderly, social visibility.

\title{
Resumo
}

A idade não deve ser considerada um elemento discriminatório para negar o acesso a educação de idosos e gerontólogos. O conhecimento faz parte dos direitos humanos: não deve haver barreiras infundadas que impeçam alguém de continuar seu desenvolvimento no ambiente escolar durante toda a vida. Este estudo avalia as oportunidades educacionais que os idosos têm. É uma investigação do tipo documentário. O quadro internacional e nacional de apoio ao idoso foi analisado; Também foi realizada uma análise do contexto atual de oportunidades educacionais em favor desse setor no México; e, por fim, foi concebida uma proposta de ação que permite a inclusão da população idosa no campo educacional. Através da educação, as pessoas idosas são fisicamente e mentalmente ativas; eles desenvolvem um senso de utilidade e pertencimento familiar e social. Certamente é um erro tratá-los como 


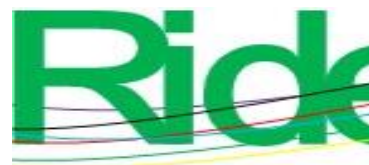

Revista Iberoamericana para la

Investigación y el Desarrollo Educativo

ISSN $2007-7467$

indivíduos passivos, sem contribuição; Como é uma posição comum nas famílias, é importante agir para erradicar esse comportamento. Os idosos têm uma riqueza de conhecimentos e experiência para aproveitar, são ativos, produtivos e podem ser incorporados em empresas e escolas por meio de programas que visam cuidar de sua participação nesses espaços e criar um ambiente digno.

Palavras-chave: direitos humanos, educação ao longo da vida, idoso, visibilidade social. Fecha Recepción: Enero 2020

Fecha Aceptación: Mayo 2020

\section{Introducción}

Todos los seres humanos poseen derechos desde su nacimiento; de ahí que se les conozca como derechos humanos. Se trata de dar a todas las personas el mismo valor y evitar la exclusión por motivos de raza, color, credo, nivel social, situación económica, nivel de estudios, estado físico, estado de salud o por edad. Es lo que se ha buscado durante mucho tiempo en todo el mundo: lograr la equidad y el trato digno en todos los ámbitos de la vida del ser humano. Sin embargo, esto no se ha logrado a plenitud, debido a que persisten varias facetas discriminatorias en la sociedad. Una de estas caras es aquella que propicia la invisibilidad de las personas en las etapas de vejez y ancianidad.

En el ámbito internacional se encuentran elementos que permiten garantizar la inclusión y el trato digno a la persona de edad, entre los que se puede mencionar: Primera Asamblea Mundial de Envejecimiento (Organización de las Naciones Unidas [ONU], 1982), Segunda Asamblea Mundial de Envejecimiento (ONU, 2002), Declaración de Toronto (Organización Mundial de la Salud [OMS], 2002), Carta de San José (Comisión Económica para América Latina y el Caribe [CEPAL], 2012), Declaración de Brasilia (CEPAL, 2011), Protocolo de San Salvador (Comisión Interamericana de Derechos Humanos [CIDH], 1988), Declaración Universal de los Derechos Humanos (ONU, 1948), Convención Interamericana sobre la Protección de los Derechos Humanos de las Personas Mayores (Organización de los Estados Americanos [OEA], 2015). Desafortunadamente, en la práctica no se cumplen. Las acciones de violación a los derechos humanos de las personas conforme tienen más edad va en aumento paulatino y sostenido. Por lo que es evidente que el problema no es la falta de leyes, ya existen suficientes, el problema es la aplicación de estas a través de los instrumentos jurídicos, aunado a la falta de seguimiento por parte de las autoridades competentes: son 


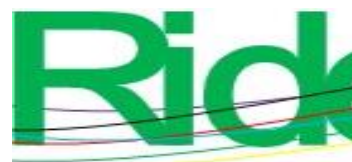

\section{Revista Iberoamericana para la Investigación y el Desarrollo Educativo ISSN $2007-7467$}

tantos los temas prioritarios en la agenda pública que pocas veces se cumple a cabalidad uno de ellos.

El Programa de las Naciones Unidas para el Desarrollo [PNUD] (2015) ha diseñado 17 objetivos para el desarrollo sostenible, que fueron presentados el 25 de septiembre de 2015: "Un llamado universal a la adopción de medidas para poner fin a la pobreza, proteger el planeta y garantizar que todas las personas gocen de paz y prosperidad" (párr. 1). A continuación, se presentan los objetivos:

1) Fin de la pobreza.

2) Hambre cero.

3) Salud y bienestar.

4) Educación de calidad.

5) Igualdad de género.

6) Agua limpia y saneamiento.

7) Energía asequible y no contaminante.

8) Trabajo decente y crecimiento económico.

9) Industria, innovación e infraestructura.

10) Reducción de las desigualdades.

11) Ciudades y comunidades sostenibles.

12) Producción y consumo responsable.

13) Acción por el clima.

14) Vida submarina.

15) Vida de ecosistemas terrestres.

16) Paz, justicia e instituciones sólidas.

17) Alianzas para lograr los objetivos (PNUD, 2015).

El objetivo cuatro expresa lo siguiente: "Lograr una educación inclusiva y de calidad para todos se basa en la firme convicción de que la educación es uno de los motores más poderosos y probados para garantizar el desarrollo sostenible" (PNUD, 2015, párr. 4). A pesar de que se encuentra enfocado en la niñez y no específicamente en las personas gerontolescentes, también está implicado el beneficio de estas últimas. Todas las naciones pertenecientes a la ONU adquirieron el compromiso de trabajar en el marco de estos 17 objetivos, por lo que sus políticas públicas deben ir encaminadas hacia su cumplimiento. 


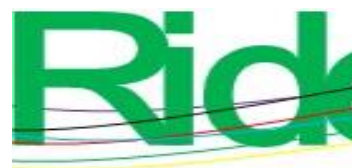

Revista Iberoamericana para la

Investigación y el Desarrollo Educativo

ISSN $2007-7467$

La Organización de las Naciones Unidas para la Educación, la Ciencia y la Cultura (UNESCO) tiene a la educación como una de sus áreas de acción prioritarias. En su quinto eje de trabajo ha considerado el aprendizaje a lo largo de la vida:

Básicamente, el aprendizaje a lo largo de la vida reposa en la integración del aprendizaje y la vida, lo que comprende actividades de aprendizaje para personas de todas las edades (niños, jóvenes, adultos y ancianos, niñas y niños, mujeres y hombres), en todos los contextos de la vida (familia, escuela, comunidad, lugar de trabajo, etc.) y mediante diversas modalidades (educación formal, no formal e informal), que en conjunto responden a una amplia gama de necesidades y exigencias relativas al aprendizaje (Instituto de la UNESCO para el aprendizaje a lo largo de toda la vida, citado en UNESCO, 2016, p. 30).

Garantizar la educación a lo largo de la vida es uno de los compromisos que tienen los secretarios de educación de los países de América Latina y el Caribe. Tomando en consideración las cifras que menciona la UNESCO (2015) de personas que no saben leer ni escribir, el problema del analfabetismo es grave. Y a pesar de las acciones que se han implementado, el resultado no se ha modificado. Por ejemplo, en México se tiene el Instituto Nacional para la Educación de los Adultos (INEA) a nivel federal y estatal, sería interesante analizar el impacto que ha tenido en estos últimos 10 años en la alfabetización a nivel nacional. Las cifras en este párrafo aludidas son las siguientes:

- 36 millones de jóvenes y adultos se reportaron como analfabetos (un $9 \%$ de la población)1, del total más de la mitad son mujeres.

- Del total de la población analfabeta, 2.5 millones están matriculados en programas de alfabetización (más de $65 \%$ son mujeres), cubriendo solo $8 \%$ de la demanda potencial siendo ciertamente insuficiente el nivel de oferta para las necesidades del continente.

- (...) Los niveles de conclusión con relación al número de participantes que inician los programas son diversos entre los países, pero en casi todos los casos es evidente la existencia de un problema serio de abandono (p. 4). 


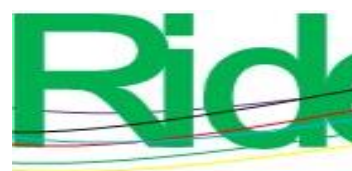

Revista Iberoamericana para la

Investigación y el Desarrollo Educativo

ISSN $2007-7467$

La forma en la que se imparte educación debe ser acorde a la edad de las personas: la educación infantil se basa en la pedagogía, la educación que se enfoca en las personas en la edad adulta se le conoce como andragogía y la forma en la que se proporciona conocimiento a las personas gerontolescentes se le llama gerontagogía o geragogía.

El siglo XXI se ha caracterizado, en parte, como el del envejecimiento de las poblaciones. De hecho, no es hasta este siglo que surge el término de personas mayores para identificar a quienes han cumplido 60 años o más (también se les conoce como adultos mayores, personas de edad o gerontolescentes). Este sector de la sociedad está llegando a la etapa conocida como tercera edad con experiencia laboral y con mejor formación académica que generaciones anteriores. Y ante esta nueva realidad no es posible excluirlos socialmente y mantenerlos en sus casas; estas personas tienen un potencial que debe ser considerado en la política educativa y aprovechado en las escuelas mexicanas.

Quienes hoy tienen 60 y más años de edad son aquellas que, en su época de juventud, lucharon por los derechos humanos. Precisamente, en el 2018 se cumplieron 70 años de la Declaración Universal de los Derechos Humanos (ONU, 1948). Como parte de la celebración de aniversario, se reafirmaron cuatro objetivos:

- Promover los derechos consagrados en la Declaración y lo que estos significan en la vida cotidiana de las personas mayores.

- Dar mayor visibilidad a las personas mayores como partes activas de la sociedad comprometidas a mejorar el goce efectivo de los derechos humanos en diversos ámbitos de la vida y no solo de aquellos que los afectan de manera inmediata.

- Reflexionar sobre el progreso y los desafíos para garantizar el disfrute pleno e igual de los derechos humanos y las libertades fundamentales de las personas de edad.

- Involucrar a un público amplio en todo el mundo y movilizar a las personas por los derechos humanos en todas las etapas de la vida. (Salud de la Junta de Castilla y León, 1 de octubre de 2018, párr. 3-6).

Los mayores defensores de los derechos humanos nacieron en el momento de la adopción de la Declaración en 1948. Entre ellos se encuentran desde personas mayores que abogan por los derechos humanos a nivel local y comunitario hasta personalidades de relieve 


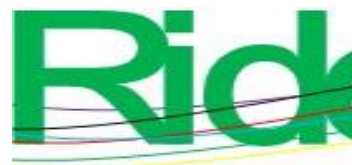

Revista Iberoamericana para la Investigación y el Desarrollo Educativo ISSN $2007-7467$

en el ámbito internacional. Todos piden el mismo respeto y reconocimiento a su dedicación y compromiso para contribuir a un mundo libre del temor y la miseria.

Tomando como referencia la observación empírica y dos entrevistas realizadas, se percibe que las nuevas generaciones de jóvenes y adultos se caracterizan por la falta de valores y una constante irreverencia hacia los ancianos, actitudes que son cada vez más comunes de observar en la sociedad actual. Sin duda ha cambiado la forma de mirar a los viejos, han quedado atrás aquellos momentos en que los ancianos eran valorados por su sabiduría y se les debía respeto y obediencia; ahora se les considera un estorbo para la familia, generadores de problemas en la cotidianidad de la casa: al ser sujetos de cuidado, los muchos gastos que implican debido a sus múltiples enfermedades, rompen con la estabilidad económica de la familia. Existe la impresión de que, al llegar a los 60 años, se llega a la edad de la invisibilidad, en donde ya no se vive, solo se espera el momento de la muerte, lo cual es un terrible error, debido a que existe una amplia gama de actividades que se pueden realizar en las etapas de la gerontolescencia, vejez y ancianidad. En la Declaración de Toronto (OMS, 2002) se reconoce el maltrato como un problema global, y se hace un llamado a la acción desde todos los ámbitos: familia, escuela, trabajo, en toda la sociedad.

En México, el Programa Sectorial de Educación 2013-2018 del Gobierno Federal (Secretaría de Educación Pública [SEP], 2013) consideró la importancia de la educación de los adultos mayores. Así quedó plasmado en la Estrategia 3. Igualdad de Oportunidades y no Discriminación contra las Mujeres: “15. Promover los programas dirigidos a adultos mayores para concluir grados escolares de la educación formal” (p. 59). Es evidente, pues, la consideración de este sector poblacional. En el Plan Nacional de Desarrollo 2019-2024 (Presidencia de la República, 2019), específicamente en el apartado Estrategia Nacional de Seguridad Pública, en el objetivo 2. Garantizar empleo, educación, salud y bienestar, se hace mención del compromiso para promover la equidad educativa en México (p. 19).

Como parte de la observación empírica, existen personas mayores de 45 años que tienen deseo de iniciar o concluir su licenciatura, la cual dejaron suspendida cuando formaron su familia, llegaron sus hijos o por falta de recurso financiero. Cuando se arman de valor y lo externan a la familia, sin embargo, reciben desmotivación y comentarios negativos; son cuestionados por la utilidad de gastar tiempo, dinero y esfuerzo. “¿Para qué? Si ya estas grande", es una de las expresiones que suelen surgir. Cuando estas personas logran vencer la presión familiar y realizan el proceso de ingreso a una institución de 


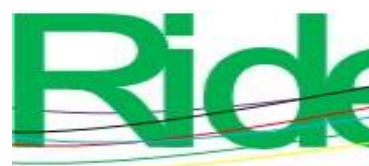

Revista Iberoamericana para la

Investigación y el Desarrollo Educativo

ISSN $2007-7467$

educación superior, los obstáculos no terminan, ya que se enfrentan a un encuentro intergeneracional en el salón de clases: la convivencia con los jóvenes, deben padecer el rechazo y las burlas por no saber bien el manejo de la tecnología e informática, la memoria comienza a fallar.

El 20 de septiembre de 2018, en el módulo gerontológico de la Universidad Autónoma de Campeche, se entrevistó a dos personas del sexo femenino que concluyeron sus estudios de licenciatura en Psicología en una escuela privada de tipo religioso. Durante la entrevista expresaron que fueron víctimas de bullying en su grupo. En un grupo de 16, había dos personas mayores, una con 50 años, casada, con dos hijos, y la otra persona con 59 años (que ahora tiene 62 años de edad), soltera y con una vida de 25 años en una congregación religiosa. Ambas se enfrentaron al rechazo de los jóvenes al momento del trabajo en equipo, en los exámenes y en las exposiciones; no faltaban las risitas burlonas, la crítica destructiva. Todo esto, no obstante, no fue suficiente para desmotivarlas y llegaron a concluir sus estudios y posteriormente lograron graduarse como licenciadas en Psicología. Esta experiencia sirve de base para este estudio en el tema de la inclusión social de las personas de edad. A través del análisis de la política educativa que actualmente desempeña el Gobierno federal, se pretende proponer una serie de acciones específicas que permitan la participación de las personas de edad en el ámbito educativo.

\section{Enfoques teóricos}

Dos son los enfoques que sustentan este estudio. La educación encierra un tesoro y Enseñanza situada: vínculo entre la escuela y la vida, a cargo de Jacques Delors (1996) y Frida Díaz Barriga (2006), respectivamente. De origen francés, Jacques Delors forma parte de la Comisión de Educación de la Unesco. En el periodo de 1985 a 1995 fue presidente de la Comisión Europea. Tiene como principal obra La educación encierra un tesoro (1996). En la cual, Delors (1996) expresa lo siguiente:

La educación durante toda la vida se presenta como una de las llaves de acceso al siglo XXI. Esta va más allá de la distinción tradicional entre educación básica y educación permanente y responde al reto de un mundo que cambia rápidamente (p. 21). 
La obra de Delors se ha mantenido vigente todos estos años. De hecho, es considerada como la base para todas las reformas educativas que se han realizado en México, debido a la vigencia de los cuatro pilares de la educación: 1) aprender a conocer, 2) aprender a hacer, 3) aprender a vivir juntos y 4) aprender a ser. Aquí se coincide con lo que afirma Delors (1996): la educación permite abrir el conocimiento para transformar nuestra realidad en un mejor escenario para disfrutar de la vida. De igual manera, expresa lo siguiente: "La formación permanente responde en gran medida a un imperativo de orden económico y permite a la empresa dotarse de personal con las mejores aptitudes, necesarias para mantener el empleo y reforzar su competitividad.” (p. 113). Es decir, que la formación continua debe mantenerse a lo largo de la vida, es la oportunidad para actualizar los conocimientos y estar en sintonía con las nuevas tendencias que se presentan. De esta forma, siempre estaremos a la altura de las nuevas generaciones. Delors (1996) también explica que "la educación a lo largo de la vida ha de brindar a cada cual los medios para alcanzar un mejor equilibrio entre el trabajo y el aprendizaje, y para el ejercicio de una ciudadanía activa” (p. 113)

Como parte de esta educación a lo largo de la vida, Delors (1996) enfatiza que la educación para las personas adultas no debe verse solamente como la alfabetización para los adultos:

La educación de adultos adopta formas múltiples, como la formación básica en un marco educativo extraescolar, la inscripción con dedicación parcial en establecimientos universitarios, los cursos de idiomas, la capacitación profesional y la actualización de conocimientos, la formación en diferentes asociaciones o sindicatos, los sistemas de aprendizaje abiertos y la formación a distancia (p. 116).

Es interesante conocer lo diverso de la oferta educativa que existe a favor de las personas gerontolescentes y de edad, partiendo del compromiso con la alfabetización de las personas. En el afán de mejorar es igualmente interesante conocer lo que se ha realizado en otros países, por ejemplo, en Suecia o Japón, donde más de 50 \% de su población adulta continúa, en un año dado, una formación estructurada, y es posible estimar que el aumento de este tipo de actividades mantiene en todo el mundo una tendencia sostenida y marcada, capaz de reorientar el conjunto de la educación en una perspectiva de educación permanente. (Delors, 1996, p. 117). Si bien estas cifras fueron presentadas hace cerca de 23 años, estos países continúan siendo un ejemplo en el rubro; a esa lista se ha añadido Alemania y España. 


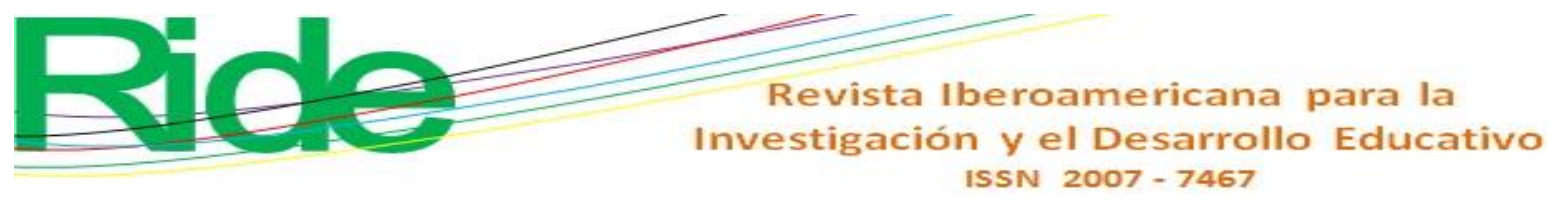

Todas estas naciones coinciden en que es un acierto ofrecer oportunidades a las personas gerontolescentes para que continúen sintiéndose útiles y ocupadas. Dichas acciones están respaldadas con base en la idea de que la educación aporta mucho en el mantenimiento de una vida con calidad.

El segundo enfoque teórico es el propuesto por Frida Díaz Barriga (2006). Mexicana, con doctorado en Pedagogía, docente en la Facultad de Psicología de la Universidad Nacional Autónoma de México (UNAM), Díaz Barriga presenta un análisis histórico de Dewey y Vigotsky. En las primeras páginas de su obra, comenta: "El conocimiento es situado, porque es parte y producto de la actividad, el contexto y la cultura en que se desarrolla y utiliza” (Díaz, 2006, p. 19). Eso es precisamente lo que se pretende en todos los niveles educativos, que el docente diseñe las secuencias didácticas teniendo como eje rector la aplicación del conocimiento para lograr que los estudiantes le encuentren a este un sentido útil y de beneficio para su vida. Díaz (2006) expresa:

No obstante que para muchos de los autores la cognición situada es la alternativa opuesta a la teoría computacional del procesamiento de información, hay distintos intereses en los investigadores de este campo, desde una orientación sociocultural y antropológica, pasando por las perspectivas ecológicas y semiótica, hasta el estudio de los procesos de cognición en diferentes planos sociales (p. 19).

Justamente lo que las personas gerontolescentes y de edad están buscando: conocimientos útiles para su vida. En este sentido, se debe construir una oferta educativa específica para este sector de la sociedad: considerarlos, hacerlos visibles, incluirlos en el diseño de las políticas públicas para lograr que verdaderamente respondan a sus necesidades.

\section{Investigaciones precedentes}

En la búsqueda de información precedente se encontraron escasos artículos relacionados con el tema de estudio. En su mayoría habla de inclusión digital o inclusión de personas con discapacidad. Teniendo en cuenta lo anterior, se seleccionaron cinco estudios como antecedentes de esta investigación: 


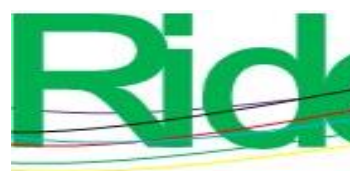

e

1

Dussan (2010) desarrolla el tema de la inclusión educativa, centrándose en las personas con discapacidad.

2) Sarrate y Pérez de Guzmán (2005) declaran que, a pesar de que en un futuro cercano habrá un mundo caracterizado por un alto porcentaje de ancianos, son muchos los países que actualmente consideran poco importante este tema.

3) Ramírez y Víctor (2010) hacen referencia al Modelo de Educación para la Vida y el Trabajo (MEVyT) que desarrolla el INEA para acompañar a las personas adultas en el proceso de adquirir su certificación de estudios de educación básica: primaria y secundaria.

4) Blázquez (2012) desarrolla la idea de que se puede aprender a cualquier edad. Y propone cambiar la visión tradicional de ver a la universidad como un espacio para formar nuevos profesionistas; ahora la universidad también debe servir para tener ciudadanos más cultos.

5) Arnay, Marrero y Fernández (2014) argumentan a favor de que las universidades permiten el desarrollo cognitivo de las personas mayores. "En el artículo se defiende una visión educativa ligada a otra manera de entender el envejecimiento, que promueva nuevas posibilidades para las personas mayores en su desarrollo intelectual, personal y social mediante su participación en la educación superior" (p. 177)

El aprendizaje a lo largo de la vida es un tema interesante de investigar sobre todo teniendo en cuenta las proyecciones demográficas de la ONU al año 2050 que indican un incremento considerable de personas mayores a los 60 años de edad (AFP, 17 de junio de 2019), a las que se les debe ofrecer actividades sociales y educativas acorde a su edad y al rol que desempeñan en las políticas públicas de México. El presente estudio es novedoso por el análisis del marco jurídico internacional, nacional y estatal, que permitió analizar lo que se ha logrado a favor de las personas mayores y proponer acciones concretas que brinden certeza a la calidad de vida de la persona de edad. Durante el desarrollo de las actividades se aplicaron las normas éticas, cuidando la integridad de las personas entrevistadas y garantizando la confidencialidad de los datos personales sensibles. El aspecto relevante de este estudio es el análisis del conjunto de normas a favor de las personas de edad y la oportunidad de la divulgación a la comunidad educativa y gerontológica. Trata de responder a la siguiente pregunta: ¿cuáles son las oportunidades que tienen las personas 


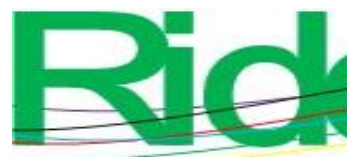

Revista Iberoamericana para la Investigación y el Desarrollo Educativo ISSN $2007-7467$

gerontolescentes? Y el objetivo, por tanto, fue evaluar las oportunidades educativas que tienen las personas de edad.

\section{Materiales y método}

Esta investigación es de tipo documental, se analizó el marco internacional y nacional que respalda a las personas de edad, así como el contexto actual de las oportunidades educativas a favor de las personas gerontolescentes en México. Al final, se formuló una propuesta de acción que permita la inclusión de las personas de edad en el ámbito educativo.

El estudio está integrado por dos etapas. En la primera se seleccionó 11 documentos (ocho son internacionales y tres nacionales), los cuales fueron analizados para identificar los aspectos que se relacionan con el tema de estudio. Los textos son: Primera Asamblea Mundial de Envejecimiento (ONU, 1982), Segunda Asamblea Mundial de Envejecimiento (ONU, 2002), Declaración de Toronto (OMS, 2002), Carta de San José (CEPAL, 2012), Declaración de Brasilia (CEPAL, 2011), Protocolo de San Salvador (CIDH, 1988), Declaración Universal de los Derechos Humanos (ONU, 1948), Convención Interamericana sobre la Protección de los Derechos Humanos de las Personas Mayores (OEA, 2015), Constitución Política de los Estados Unidos Mexicanos (Cámara de Diputados del H. Congreso de la Unión, 2019), Ley de los Derechos de las Personas Adultas Mayores (Cámara de Diputados del H. Congreso de la Unión, 2018) y Plan Nacional de Desarrollo 2019-2024 (Presidencia de la República, 2019).

En la segunda etapa se realizó la revisión de la oferta de convocatorias de ingreso al nivel superior de 10 instituciones públicas para identificar si existen restricciones por edad; también se analizaron las actividades que realiza el INEA en México; y por último, se realizó un análisis de los programas universitarios para mayores con el fin de conocer tanto las actividades que ofrecen como los requisitos y las generalidades de esta oferta para las personas en las etapas de madurez y ancianidad. El período de recolección y selección de la información fue de febrero a agosto de 2019.

Respecto a la población y muestra del estudio, no se trabajó con personas (sujetos) debido a que el estudio es de corte documental, en la línea del análisis de documentos vigentes a favor de las personas gerontolescentes. 


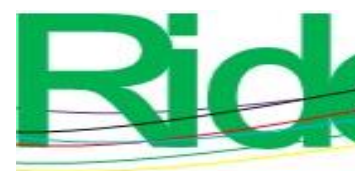

Revista Iberoamericana para la

Investigación y el Desarrollo Educativo ISSN $2007-7467$

\section{Resultados}

De los 11 documentos seleccionado, 8 son internacionales y 3 mexicanos. En conjunto abarcan los años de 1948 a 2019. A continuación, un recorrido por los más representativos documentos internacionales a favor de las personas de edad. En primer lugar, la Declaración Universal de los Derechos Humanos (ONU, 1948), en su artículo 26, considera el derecho a la educación. La Primera Asamblea Mundial de Envejecimiento (ONU, 1982) coincide en la preocupación sobre el envejecimiento. Además, traza los cimientos a partir de dos líneas: en el aspecto de salud, con la educación en el autocuidado y la gerontologización del personal del área de la salud; el otro aspecto es la educación a lo largo de la vida, sin discriminación, considerando no solo la alfabetización, sino el nivel universitario, además de la importancia de gerontologizar a la sociedad.

El Protocolo de San Salvador (CIDH, 1988) se suma a la lucha contra la discriminación y empatiza con la protección a los ancianos y su derecho a la educación. En la Segunda Asamblea Mundial de Envejecimiento (ONU, 2002), por su parte, se reflexiona sobre la necesidad de elevar el nivel educativo de las personas de edad en los países en vías de desarrollo, con el fin de tener un mejor nivel de vida, a través de igualdad de oportunidades, de capacitación en temas de informática y actividades técnicas que puedan realizar en la etapa de su jubilación. En la Declaración de Toronto (OMS, 2002) se reconoce el maltrato como problema global y la necesidad de trabajar para erradicar el limitado marco legal existente. La Declaración de Brasilia (CEPAL, 2011) parte del hecho de que el envejecimiento puede causar dependencia y se une al llamado de permitir el acceso a la educación continua, así como al de crear programas educativos en gerontología social. En la Carta de San José (CEPAL, 2012) el problema de la discriminación hacia las personas mayores resulta evidente, y se une a las voces que han insistido en la educación permanente, al igual que la gerontologización desde edades tempranas. De forma análoga, en la Convención Interamericana sobre la Protección de los Derechos Humanos de las Personas Mayores (OEA, 2015) se insiste en la necesidad de combatir la discriminación, el derecho a la educación sin importar la edad y la necesidad de gerontologizar. Estos ocho documentos están unidos por los temas del derecho a la educación a lo largo de la vida, la lucha contra la discriminación por razones de edad y la necesidad de gerontologizar desde la niñez (ver anexo, tabla 1). 


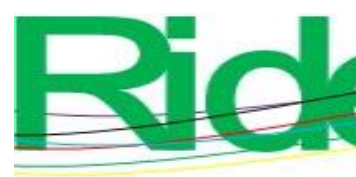

Revista Iberoamericana para la Investigación y el Desarrollo Educativo ISSN $2007-7467$

En lo que respecta al análisis de los tres documentos nacionales, se identificó que igualmente coinciden en el derecho a la educación para las personas mayores. Primeramente, la Constitución Política de los Estados Unidos Mexicanos (Cámara de Diputados del H. Congreso de la Unión, 2019), en su artículo 3, declara el derecho a la educación de todos los mexicanos. Seguidamente, la Ley de los Derechos de las Personas Adultas Mayores (Cámara de Diputados del H. Congreso de la Unión, 2018) otorga a este grupo de la sociedad autonomía, los defiende de la discriminación y garantiza su derecho al acceso a la educación en todos los niveles, además de enfatizar en la necesidad de la gerontologización desde la niñez. Por último, en el Plan Nacional de Desarrollo 2019-2024 (Gobierno de la República, 2019) considera que la educación y la salud son necesarias para promover la equidad educativa.

En la revisión de la oferta de convocatorias de ingreso al nivel superior en la república mexicana, se encontraron y analizaron 10 convocatorias de ingreso al nivel superior, publicadas en el mes de febrero de 2019. A partir del análisis, se comprobó que no existe restricción por motivo de edad; al menos en la convocatorio no se identificaron rasgos de discriminación. Esta oferta tiene la intención de realizar un encuentro intergeneracional, al propiciar la convivencia de jóvenes universitarios con personas mayores dentro del aula. Este hecho habla de un alto nivel de madurez: jóvenes y gerontolescentes conviven en un marco de paciencia y respeto.

Las actividades que se realizan en el INEA en México tienen la intención de abatir el rezago educativo con la alfabetización. En 2015 se tenía cerca de $50 \%$ de rezago en el grupo de personas entre 50 y 55 años de edad. Es un tema en el que se continúa trabajando para que las futuras generaciones de viejos tengan mejor calidad de vida.

En México existen instituciones de educación superior que ofrecen programas universitarios para mayores. Aquí se revisaron cuatro de estas: Benemérita Universidad Autónoma de Puebla, Universidad Iberoamericana Puebla, Universidad de Ecatepec y Universidad Autónoma de Yucatán. Los programas que ofrecen son para desarrollar aspectos culturales, de calidad de vida personal y social que incidan en su desarrollo humano. Este tipo de propuesta educativa trata de propiciar la socialización entre iguales. Es decir, solo acuden personas mayores, quienes reciben instrucción formal por parte de otra persona, esto es más común y solo se obtiene una constancia de participación por módulo, si se 

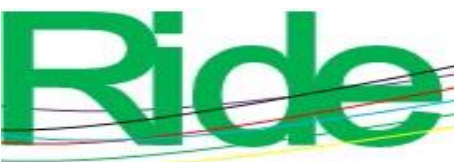

Revista Iberoamericana para la

Investigación y el Desarrollo Educativo

ISSN $2007-7467$

acreditan varios módulos tienen la posibilidad de aspirar a un diploma; no se consideran los créditos académicos suficientes para optar por un título o grado académico.

\section{Discusión}

A partir de todo lo anterior, se comprueba lo expresado por Delors (1996): "La educación a lo largo de la vida ha de brindar a cada cual los medios para alcanzar un mejor equilibrio entre el trabajo y el aprendizaje, y para el ejercicio de una ciudadanía activa" (p. 113). Indudablemente el escenario ha cambiado, ha quedado atrás la meta de alfabetizar a los adultos, ahora la prioridad es mantener la habilidad cognitiva de las personas y la propuesta es que se realice a través de la vía de la educación a lo largo de la vida.

Una de las limitaciones de este estudio es el análisis de los documentos, debido a que no se logró cubrir el total de información que actualmente existe, y se recurrió a una selección determinada. Otra limitante fue la selección de las universidades que tienen el desarrollo de los programas universitarios para mayores. Únicamente se ubicaron las opciones que aparecieron en Internet; hace falta enriquecer la búsqueda con otros medios más académicos.

Entre las fortalezas de este artículo está la integración de experiencias de vida que han tenido dos personas gerontolescentes al regresar a la escuela, con la ilusión de concluir sus estudios de licenciatura. La principal fortaleza, sin embargo, es que en este documento reúne elementos importantes que otros autores han desarrollado de forma individual, como son: el análisis de documentos jurídicos relacionados con los derechos de las personas mayores, la descripción de los programas universitarios para mayores y el derecho a la educación a lo largo de la vida, que debe continuar a pesar de la edad de las personas. 


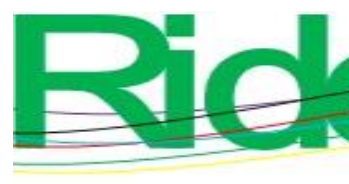

Revista Iberoamericana para la

Investigación y el Desarrollo Educativo

ISSN $2007-7467$

\section{Conclusiones}

Todos los documentos analizados consideran trascendental el derecho a la educación y la lucha contra la discriminación de las personas mayores, así como la necesidad de gerontologizar al personal del área de la salud y a la sociedad en general desde edades tempranas.

Las personas gerontolescentes tienen las siguientes oportunidades educativas:

1) Continuar y concluir con su formación profesional formal en una institución de educación superior, conviviendo con las nuevas generaciones, lo cual es importante para mantener su desarrollo cognitivo y sus habilidades sociales, con la esperanza de obtener un título universitario.

2) Participar en los cursos que ofrecen los programas universitarios para mayores; cursos estructurados, enfocados en el desarrollo humano, cuya intención es la socialización entre pares para generar una red de apoyo.

3) Las personas mayores que son analfabetas tienen la oportunidad de acceder a los cursos gratuitos que ofrece el INEA.

Este estudio alcanza su objetivo al presentar el análisis de las opciones educativas que tienen las personas de edad. La educación es la oportunidad que tienen las personas mayores de mantener su actividad física y mental, de sentirse vivos, útiles y parte de una sociedad, de no perder visibilidad en su familia.

La propuesta para la inclusión de las personas gerontolecentes está integrada por dos dimensiones. La primera es empoderarlos; iniciar con un diagnóstico que permita identificar sus fortalezas, oportunidades, debilidades y amenazas (análisis FODA), lo cual permitirá identificar sus habilidades y áreas de experiencia. La segunda es vincularlos con el área educativa a través de dos ejes: 1) aportación social a las nuevas generaciones y 2) inclusión universitaria. En el primero se trata de expandir las escuelas de todos los niveles para permitir que estas personas, que a partir de ahora se denominarán con talento oro, puedan tener un espacio mensual en el que, a través de una charla directa con los estudiantes, se compartan experiencias o asesoría sobre temas académicos específicos. En el segundo, las personas con talento oro tendrán acceso a las instituciones de educación superior para tomar cursos que respondan a sus necesidades y en beneficio de su desarrollo biopsicosocial. 


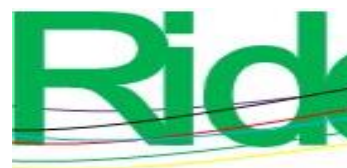

Revista Iberoamericana para la Investigación y el Desarrollo Educativo ISSN $2007-7467$

Es un error tratar a las personas mayores como personas pasivas, que ya no pueden aportar nada. Esta es una postura que cada vez es más común en las familias, por lo que es importante que se actúe para erradicarla. Las personas mayores tienen un cúmulo de conocimientos y experiencia que debe ser aprovechado por las nuevas generaciones. Tienen mucho que aportar a la familia y sociedad. Son personas activas, que pueden ser todavía productivas, deben ser incorporadas a las empresas y a las escuelas a través de programas destinados a cuidar la participación de las personas gerontolescentes en estos espacios, en un ambiente dignificado. Las personas que en esta segunda década del siglo XXI están llegando a los 70 años de edad son totalmente distintas; ahora se caracterizan por tener estudios universitarios concluidos y una amplia experiencia laboral, y desean mantener su habilidad cognitiva a través de la inclusión social. Paulatinamente se irán añadiendo personas ancianas con estudios de posgrados y amplia experiencia en investigación, en consecuencia, es importante que las políticas públicas consideren este contexto socio demográfico, para que, a través del sistema educativo y sociedad, los incluya en las acciones que permitan prolongar la utilidad de las personas viejas, lo cual incidirá en el mejoramiento de su calidad de vida y si tienen calidad de vida, se enfermarán menos, disminuyendo la carga social que representa un país de ancianos pluripatológicos. 


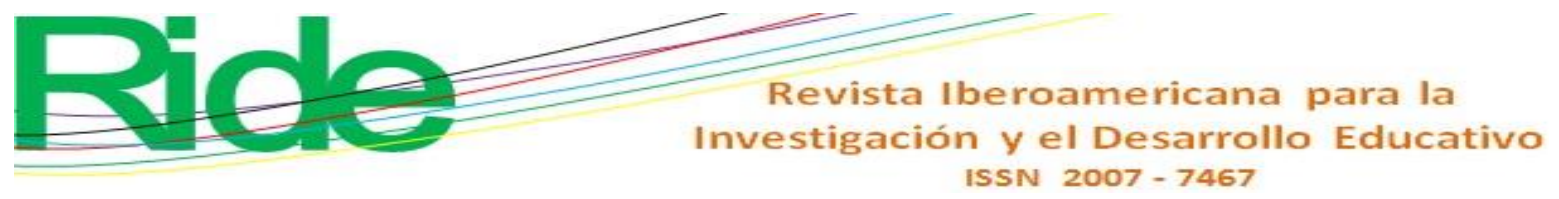

\section{Referencias}

Arnay, J., Marrero, J. y Fernández, I. (2012) Las universidades para mayores: ¿qué enseñanza, qué aprendizaje? Memorialidades, 9(17), 177-213. Recuperado de https://periodicos.uesc.br/index.php/memorialidades/article/view/61.

Cámara de Diputados del H. Congreso de la Unión. (2018) Ley de los Derechos de las Personas Adultas Mayores. México: Cámara de Diputados del H. Congreso de la Unión. Recuperado

de http://www.diputados.gob.mx/LeyesBiblio/pdf/245_120718.pdf.

Cámara de Diputados del H. Congreso de la Unión. (2019) Constitución Política de los Estados Unidos Mexicanos. México: Cámara de Diputados del H. Congreso de la Unión.

Recuperado

de http://www.diputados.gob.mx/LeyesBiblio/pdf/1_090819.pdf.

Comisión Económica para América Latina y el Caribe [Cepal]. (2011). Declaración de Brasilia: Segunda Conferencia Regional Intergubernamental sobre Envejecimiento en América Latina y el Caribe: hacia una sociedad para todas las edades y de protección social basada en derechos. Brasilia, Brasil: Comisión Económica para América Latina y el Caribe. Recuperado de https://www.cepal.org/es/publicaciones/21505-declaracion-brasilia-segundaconferencia-regional-intergubernamental

Comisión Económica para América Latina y el Caribe [Cepal]. (2012). Carta de San José sobre los derechos de las personas mayores de América Latina y el Caribe. San José, Costa Rica: Comisión Económica para América Latina y el Caribe. Recuperado de https://www.cepal.org/celade/noticias/paginas/1/44901/CR_Carta_ESPÁG.pdf.

Comisión Interamericana de Derechos Humanos [CIDH]. (1988). Protocolo de San Salvador. San Salvador, El Salvador: Comisión Interamericana de Derechos Humanos. Recuperado de https://catedraunescodh.unam.mx/catedra/pronaledh/indexbfd2.html?option=com_c ontent $\&$ view $=$ article $\& i d=43$ :protocolo-san-salvador $\&$ catid $=13 \&$ Itemid $=26$.

Delors, J. (1996). La educación encierra un tesoro. Madrid, España: Unesco. Recuperado de http://innovacioneducativa.uaem.mx:8080/innovacioneducativa/web/Documentos/e ducacion_tesoro.pdf. 


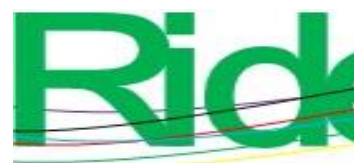

Revista Iberoamericana para la

Investigación y el Desarrollo Educativo

ISSN $2007-7467$

Díaz, F. (2006). Enseñanza situada: vínculo entre la escuela y la vida. México: McGrawHill.

Gobierno de la República, 2019. Plan Nacional de Desarrollo 2019 - 2024. Diario Oficial de la Federación. Recuperado de https://www.dof.gob.mx/nota_detalle.php?codigo=5565599\&fecha=12/07/2019

Organización de Estados Americanos [OEA]. (2015). Convención Interamericana sobre la Protección de los Derechos Humanos de las Personas Mayores (A-70). Organización de Estados Americanos. Recuperado de http://www.oas.org/es/sla/ddi/tratados_multilaterales_interamericanos_a70_derechos_humanos_personas_mayores.asp.

Organización de las Naciones Unidas [ONU]. (1948). Declaración Universal de los Derechos Humanos. Organización de las Naciones Unidas. Recuperado de https://www.un.org/es/universal-declaration-human-rights/.

Organización de las Naciones Unidas [ONU]. (1982). Primera Asamblea Mundial de Envejecimiento. Plan de acción internacional de Viena sobre el envejecimiento. Viena, Austria: Organización de las Naciones Unidas. Recuperado de http://www.mayoressaludables.org/sites/default/files/2017-

03/plan_de_accion_internacional_de_viena_sobre_el_envejecimiento.pdf

Organización de las Naciones Unidas [ONU]. (2002). Segunda Asamblea Mundial de Envejecimiento. Madrid, España: Organización de las Naciones Unidas. Recuperado de https://www.un.org/es/events/pastevents/ageing_assembly2/ .

Organización de las Naciones Unidas para la Educación, la Ciencia y la Cultura [UNESCO] (2016). Declaración de Incheon. Organización de las Naciones Unidas para la Educación, la Ciencia y la Cultura. Recuperado de https://unesdoc.unesco.org/ark:/48223/pf0000245656_spa.

Organización Mundial de la Salud [OMS]. (2002) Declaración de Toronto. Ginebra, Suiza: Organización Mundial de la Salud. Recuperado de http://www.inpea.net/images/TorontoDeclaracion_Espanol.pdf.

Presidencia de la República. (2019). Plan Nacional de Desarrollo 2019-2024. México: Presidencia de la República. Recuperado de https://lopezobrador.org.mx/wpcontent/uploads/2019/05/PLAN-NACIONAL-DE-DESARROLLO-2019-2024.pdf. 
Programa de las Naciones Unidas para el Desarrollo [PNUD]. (2015). Objetivos de Desarrollo Sostenible. Nueva York, Estados Unidos: Programa de las Naciones Unidas para el Desarrollo. Recuperado de https://www.undp.org/content/undp/es/home/sustainable-development-goals.html.

Salud de la Junta de Castilla y León. (1 de octubre de 2018). Día Internacional de las Personas de Edad. proxia suite. Recuperado de https://www.saludcastillayleon.es/AulaPacientes/en/dias-mundiales-relacionadossalud/dia-internacional-personas-edad-150e53.print-frame.

Secretaría de Educación Pública [SEP]. (2013) Programa Sectorial de Educación 2013-2018. México: Secretaría de Educación Pública. Recuperado de https://www.gob.mx/cms/uploads/attachment/file/11908/PROGRAMA_SECTORI AL_DE_EDUCACION_2013_2018_WEB.compressed.pdf. 


\section{Anexo}

Tabla 1. Concentrado del marco legal que respalda a la persona gerontolescente

\begin{tabular}{|c|c|c|}
\hline Documento & Datos generales & Aportación \\
\hline $\begin{array}{l}\text { Declaración } \\
\text { Universal de los } \\
\text { Derechos } \\
\text { Humanos }\end{array}$ & $\begin{array}{l}10 \text { diciembre } 1948 . \\
\text { ONU. }\end{array}$ & $\begin{array}{l}\text { Artículo 1. Todos los seres humanos } \\
\text { nacen libres. } \\
\text { Artículo 7. Protección contra la } \\
\text { discriminación. } \\
\text { Artículo 26. Derecho a la educación. }\end{array}$ \\
\hline $\begin{array}{l}\text { Primera } \\
\text { Asamblea } \\
\text { Mundial de } \\
\text { Envejecimiento }\end{array}$ & $\begin{array}{l}\text { Llevada a cabo del } 26 \\
\text { julio al } 6 \text { agosto de } \\
1982 \text { en Viena, Austria. }\end{array}$ & $\begin{array}{l}\text { Preocupación común sobre } \\
\text { envejecimiento. } \\
\text { Recomendación 7: } \\
\text { - Deberá educarse a las propias personas } \\
\text { de edad en el cuidado de sí mismas. } \\
\text { - Capacitar al personal del área de la } \\
\text { salud en temas de gerontología, } \\
\text { geriatría y psicogeriatría. (p. 24) } \\
\text { Recomendación 45: } \\
\text { La educación debe proporcionarse sin } \\
\text { discriminación contra las personas de } \\
\text { edad. } \\
\text { La necesidad de la educación continua } \\
\text { de adultos a todos los niveles. } \\
\text { Habrá de tomarse en consideración el } \\
\text { concepto de la educación universitaria } \\
\text { para personas de edad (p. 33) } \\
\text { Recomendación } 76 \\
\text { Educar a la población en general acerca } \\
\text { del proceso de envejecimiento (p. 33) } \\
\text { Recomendación } 54 \\
\text { Los programas de enseñanza y } \\
\text { capacitación deberán ser de carácter } \\
\text { interdisciplinario. } \\
\text { La enseñanza y capacitación en los } \\
\text { diversos aspectos del envejecimiento } \\
\text { deberá impartirse a todos los niveles. (p. } \\
36 \text { ) }\end{array}$ \\
\hline $\begin{array}{l}\text { Protocolo de San } \\
\text { Salvador }\end{array}$ & $\begin{array}{l}\text { 1988. Adicional a la } \\
\text { Convención Americana } \\
\text { sobre Derechos } \\
\text { Humanos en Materia de } \\
\text { Derechos Económicos, } \\
\text { Sociales y Culturales. }\end{array}$ & $\begin{array}{l}\text { Artículo 3. Obligación de no } \\
\text { discriminación. } \\
\text { Artículo 13. Derecho a la educación. } \\
\text { Artículo 17. Protección a los ancianos }\end{array}$ \\
\hline
\end{tabular}




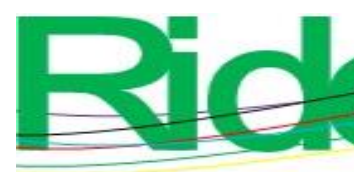

Revista Iberoamericana para la

Investigación y el Desarrollo Educativo ISSN 2007 - 7467

\begin{tabular}{|c|c|c|}
\hline $\begin{array}{l}\text { Segunda } \\
\text { Asamblea } \\
\text { Mundial de } \\
\text { Envejecimiento }\end{array}$ & 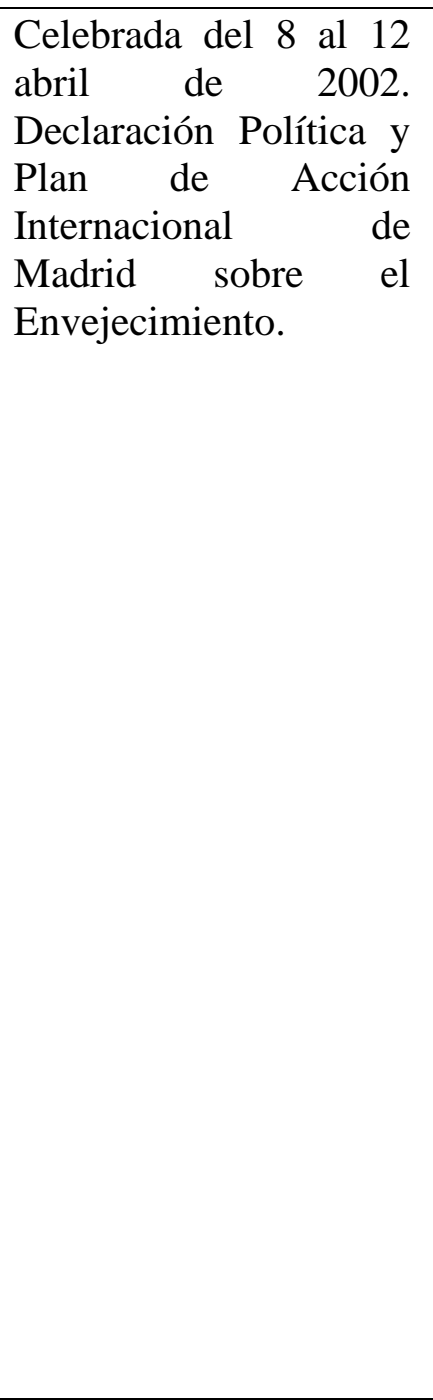 & 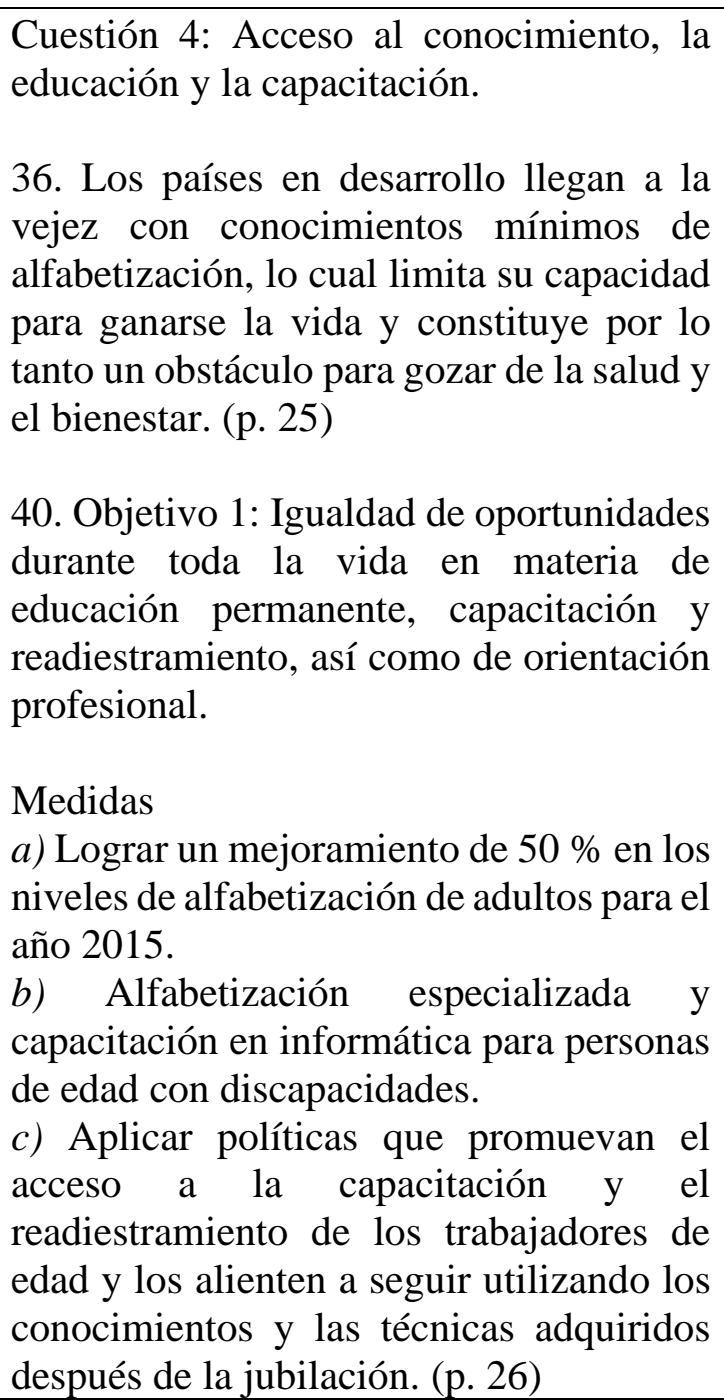 \\
\hline $\begin{array}{ll}\text { Declaración } & \text { de } \\
\text { Toronto para la } \\
\text { Prevención } \\
\text { Global del } \\
\text { Maltrato de las } \\
\text { Personas } & \\
\text { Mayores } & \\
\end{array}$ & 2002. OMS & $\begin{array}{l}\text { - Reconoce el maltrato como un } \\
\text { problema global. (párr. 1) } \\
\text { - Llamado a la acción dirigida a la } \\
\text { prevención del maltrato de las personas } \\
\text { mayores. (párr. 5) } \\
\text { - Reconoce la falta de marcos legales. } \\
\text { (párr. 6) }\end{array}$ \\
\hline $\begin{array}{l}\text { Declaración de } \\
\text { Brasilia }\end{array}$ & $\begin{array}{l}\text { Segunda Conferencia } \\
\text { Regional sobre } \\
\text { Envejecimiento en } \\
\text { América Latina y el } \\
\text { Caribe: hacia una } \\
\text { sociedad para todas las } \\
\text { edades y de protección } \\
\text { social basada en } \\
\text { derechos. Llevada a } \\
\text { cabo del } 4 \text { al } 6 \\
\text { diciembre de } 2011 .\end{array}$ & $\begin{array}{l}\text { - Identifica prioridades futuras. (p. 5) } \\
\text { - Se mantiene la brecha en la } \\
\text { implementación de los derechos. (p. 5) } \\
\text { - Se reconoce que el envejecimiento } \\
\text { puede generar discapacidades y } \\
\text { dependencia. (p. 6) } \\
\text { - Impulsa el acceso a la educación } \\
\text { continua y permanente. (p. 9) } \\
\text { - Propicia crear programas de } \\
\text { licenciatura y maestría en gerontología } \\
\text { social. (p. 9) }\end{array}$ \\
\hline
\end{tabular}




\begin{tabular}{|c|c|c|}
\hline $\begin{array}{l}\text { Carta de San José } \\
\text { sobre los } \\
\text { Derechos de las } \\
\text { Personas } \\
\text { Mayores de } \\
\text { América Latina y } \\
\text { el Caribe }\end{array}$ & $\begin{array}{l}\text { Tercera Conferencia } \\
\text { Regional sobre } \\
\text { Envejecimiento en } \\
\text { América Latina y el } \\
\text { Caribe. Llevada a cabo } \\
\text { del } 8 \text { al } 11 \text { mayo de } \\
2012 \text {. }\end{array}$ & $\begin{array}{l}\text { - Identifica acciones clave en derechos } \\
\text { humanos y protección social a las } \\
\text { personas mayores. (p. 5) } \\
\text { - Confirma la existencia de } \\
\text { discriminación en las personas } \\
\text { mayores. (p. 5) } \\
\text { - Insiste en la educación permanente y } \\
\text { promueve incluir el tema de } \\
\text { envejecimiento desde la niñez. (p. 15) }\end{array}$ \\
\hline $\begin{array}{l}\text { Convención } \\
\text { Interamericana } \\
\text { sobre la } \\
\text { Protección de los } \\
\text { Derechos } \\
\text { Humanos de las } \\
\text { Personas } \\
\text { Mayores }\end{array}$ & 2015. OEA & $\begin{array}{l}\text { Instrumento regional jurídicamente } \\
\text { vinculante. } \\
\text { Artículo 5. Igualdad y no discriminación } \\
\text { por razones de edad. (párr. 40) } \\
\text { Artículo 20. Derecho a la educación. (párr. } \\
\text { 113) } \\
\text { Artículo 32 } \\
\text { c) Desarrollar programas para sensibilizar } \\
\text { a la población sobre el proceso de } \\
\text { envejecimiento. (párr. 163) }\end{array}$ \\
\hline $\begin{array}{l}\text { Ley de los } \\
\text { Derechos de las } \\
\text { Personas Adultas } \\
\text { Mayores }\end{array}$ & $\begin{array}{l}\text { 2018. Cámara de } \\
\text { Diputados del H. } \\
\text { Congreso de la Unión. }\end{array}$ & $\begin{array}{l}\text { Principios rectores: autonomía y } \\
\text { autorrealización, participación, equidad, } \\
\text { corresponsabilidad y atención preferente. } \\
\text { Artículo 5 } \\
\text { IV. Derecho a la educación. Incluir en sus } \\
\text { planes y programas los conocimientos } \\
\text { relacionados con las personas adultas } \\
\text { mayores; asimismo los libros de texto } \\
\text { gratuitos y todo material educativo, } \\
\text { incorporarán información sobre el tema } \\
\text { del envejecimiento y las personas adultas } \\
\text { mayores (p. 5). } \\
\text { Artículo 8. Ninguna persona adulta mayor } \\
\text { podrá ser socialmente marginada o } \\
\text { discriminada en ningún espacio público o } \\
\text { privado (p. 7). } \\
\text { Artículo 17. Corresponde a la Secretaría } \\
\text { de Educación Pública, garantizar a las } \\
\text { personas adultas mayores el acceso a la } \\
\text { educación pública en todos sus niveles y } \\
\text { modalidades y a cualquier otra actividad } \\
\text { que contribuya a su desarrollo intelectual } \\
\text { y que le permita conservar una actitud de } \\
\text { aprendizaje constante y aprovechar toda } \\
\text { oportunidad de educación y capacitación } \\
\text { que tienda a su realización personal, } \\
\text { facilitando los trámites administrativos y }\end{array}$ \\
\hline
\end{tabular}




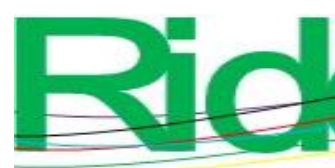

Revista Iberoamericana para la

\begin{tabular}{|l|l|l|}
\hline & & $\begin{array}{l}\text { difundiendo la oferta general educativa (p. } \\
10) .\end{array}$ \\
\hline $\begin{array}{l}\text { Constitución } \\
\text { Política de los } \\
\text { Estados Unidos } \\
\text { Mexicanos }\end{array}$ & $\begin{array}{l}\text { 2019. Cámara de } \\
\text { Diputados del H. } \\
\text { Congreso de la Unión. }\end{array}$ & $\begin{array}{l}\text { Artículo 3. Toda persona tiene derecho a } \\
\text { la educación. (p. 5) } \\
\text { Corresponde al Estado la rectoría de la } \\
\text { educación, además de obligatoria, será } \\
\text { universal, inclusiva, pública, gratuita y } \\
\text { laica. } \\
\text { La educación se basará en el respeto } \\
\text { irrestricto de la dignidad de las personas, } \\
\text { con un enfoque de derechos humanos y de } \\
\text { igualdad sustantiva (p. 5). }\end{array}$ \\
\hline $\begin{array}{l}\text { Plan Nacional de } \\
\text { Desarrollo 2019- } \\
\text { 2024 }\end{array}$ & $\begin{array}{l}\text { 2019. Gobierno de la } \\
\text { República }\end{array}$ & $\begin{array}{l}\text { Objetivo 2. Garantizar empleo, educación, } \\
\text { salud y bienestar, la cual está enfocada en } \\
\text { los jóvenes } \\
\text { Evidenciando el aspecto asistencialista } \\
\text { con la Pensión Universal para Personas } \\
\text { Adultas Mayores. (párr. 50) }\end{array}$ \\
\hline
\end{tabular}

Fuente: Elaboración propia, marzo 2020 